\title{
Chemical abundances of massive stars in Local Group galaxies
}

\author{
Kim A. Venn ${ }^{1}$, Andreas Kaufer ${ }^{2}$, Eline Tolstoy ${ }^{3}$, Rolf-Peter Kudritzki ${ }^{4}$, \\ Norbert Przybilla ${ }^{4}$, Stephen J. Smartt ${ }^{5}$, and Daniel J. Lennon ${ }^{6}$ \\ ${ }^{1}$ University of Minnesota and Macalester College, \\ 1600 Grand Avenue, Saint Paul, MN 55105, USA \\ ${ }^{2}$ European Southern Observatory, \\ Alonso de Cordova 3107, Santiago 19, Chile \\ ${ }^{3}$ Kapteyn Instituut, Universiteit Groningen, \\ Postbus 800, NL-9700 AV Groningen, Nederland \\ ${ }^{4}$ Institute for Astronomy, University of Hawaii at Manoa, \\ 2680 Woodlawn Dr., Honolulu, HI 96822, USA \\ ${ }^{5}$ Institute of Astronomy, University of Cambridge, \\ Madingley Road, Cambridge, CB30HA, UK \\ ${ }^{6}$ Isaac Newton Group of Telescopes, Apartado de correos 321, \\ Santa Cruz de La Palma, Tenerife, E-38700, España
}

\begin{abstract}
The relative abundances of elements in galaxies can provide valuable information on the stellar and chemical evolution of a galaxy. While nebulae can provide abundances for a variety of light elements, stars are the only way to directly determine the abundances of iron-group and $s$-process and $r$-process elements in a galaxy. The new $8 \mathrm{~m}$ and $10 \mathrm{~m}$ class telescopes and high-efficiency spectrographs now make high-quality spectral observations of bright supergiants possible in dwarf galaxies in the Local Group. We have been concentrating on elemental abundances in the metal-poor dwarf irregular galaxies, NGC 6822, WLM, Sextants A, and GR 8. Comparing abundance ratios to those predicted from their star formation histories, determined from color-magnitude diagrams, and comparing those ratios between these galaxies can give us new insights into the evolution of these dwarf irregular galaxies. Iron-group abundances also allow us to examine the metallicities of the stars in these galaxies directly, which affects their inferred mass loss rates and predicted stellar evolution properties.
\end{abstract}

\section{Introduction}

A map of the Local Group shows that most dwarf irregular galaxies are relatively isolated systems beyond the expected dark matter halos of the large spiral galaxies (e.g., Grebel 1999). Nebular analyses of the gas phase abundances in these galaxies show that they are metal-poor systems (e.g., Pagel, Edmunds \& Smith 1980; Skillman et al. $1989 \mathrm{a}, \mathrm{b})$. With the new 8 to $10 \mathrm{-m}$ class telescopes and high efficiency spectrographs, it is now possible to determine elemental abundances from the brightest stars in these galaxies. The visually brightest stars are the blue supergiants. Recent analyses of A-type supergiants in the Galaxy, the 
SMC, and NGC 6822 (Venn 1995, 1999, Venn et al. 2001; Przybilla et al. 2001) have shown that many more elements can be observed than from nebular analyses; particularly, iron-group and some $s \& r$-process elements. It is important to ascertain the metallicities, and detailed elemental abundances, in these stars since metals affect the inferred mass loss rates as well as the evolution of the stars. But in addition, the element ratios can also tell us about the chemical evolution of these dwarf galaxies.

In this paper, I will summarize our preliminary abundance results from analyses of stars in NGC 6822, WLM, Sextants A and GR 8. These abundances will be compared to nebular results, and put into the context of the star formation histories of these galaxies as predicted from their color-magnitude diagrams.

\section{2. $\quad$ NGC 6822}

Examination of the color-magnitude diagram for NGC 6822 (Gallart et al. 1996a) shows several blue supergiants that are 1 to 2 magnitudes above the tip of the red giant branch. We have obtained spectra for five bright A-type supergiants from the VLT-UVES, as well as observations for two stars with KECK-HIRES. Three stars have been analysed (results for two of these stars are in Venn et al. 2001). The average oxygen abundance for these three stars is in excellent agreement with the nebular result, $[\mathrm{O} / \mathrm{H}] \simeq-0.5$ (Pagel et al. 1980; Pilyugin 2001; Skillman et al. 1989b). From the stars, we now also know that iron, and most of the other elements analysed, have the same underabundance, i.e., $[\mathrm{Fe} / \mathrm{H}] \simeq-0.5$.

Figure 1 shows the average abundances for the three A-type supergiants analysed in NGC 6822, and for comparison the average abundances from ten stars analysed in the SMC. Clearly, NGC 6822 is slightly more metal-rich than the SMC, but both galaxies are metal-poor relative to the Sun. Also, both NGC 6822 and the SMC show the same underabundances of $\alpha$-elements (even-Z light elements; $\mathrm{O}, \mathrm{Mg}, \mathrm{Si}$, and possibly $\mathrm{Ti}$ ) as for iron-group elements $(\mathrm{Cr}, \mathrm{Fe}$, and possibly $\mathrm{Ti}$ ). Only the $s \& r$-process element $\mathrm{Sr}$ deviates significantly from this pattern, with very strong underabundances in both galaxies.

$\alpha / \mathrm{Fe}$ ratios: Firstly, a discussion of the $\alpha$-element and $\mathrm{Fe}$ abundances. In studies of the Galactic halo, the $\alpha / \mathrm{Fe}$ ratio is taken as an indicator of the relative fraction of gas from SN II to that from SN Ia (see McWilliam 1997). It is striking that the $\alpha / \mathrm{Fe}$ ratios are so similar between these two galaxies considering the extreme differences in their predicted SFHs. Gallart et al. (1996b) have deduced the SFH in NGC 6822 from a detailed analysis of its color-magnitude diagram. Their figure 12 suggests a fairly steady low star formation rate between 15 and $5 \mathrm{Gyr}$, followed by a relative hiatus in star formation until sometime $<1 \mathrm{Gyr}$ ago when the current strong burst of star formation began. In contrast, the SFH in the SMC, analysed by Pagel \& Tautvaišiene (1998), has two possibilities based on the numerous abundance studies of young and old stars. Either the star formation has been fairly smooth and continuous over the past $15 \mathrm{Gyr}$, or it has had a bursting history, with a small burst around $13 \mathrm{Gyr}$ ago, followed by a relative hiatus, and a more substantial burst that started $4 \mathrm{Gyr}$ ago (see their figure 2). Considering the orbital interactions of the SMC with the LMC and the Galaxy, the bursting history is often favored. Nevertheless, both of these SFHs for the SMC are significantly different from that for NGC 6822, and the 


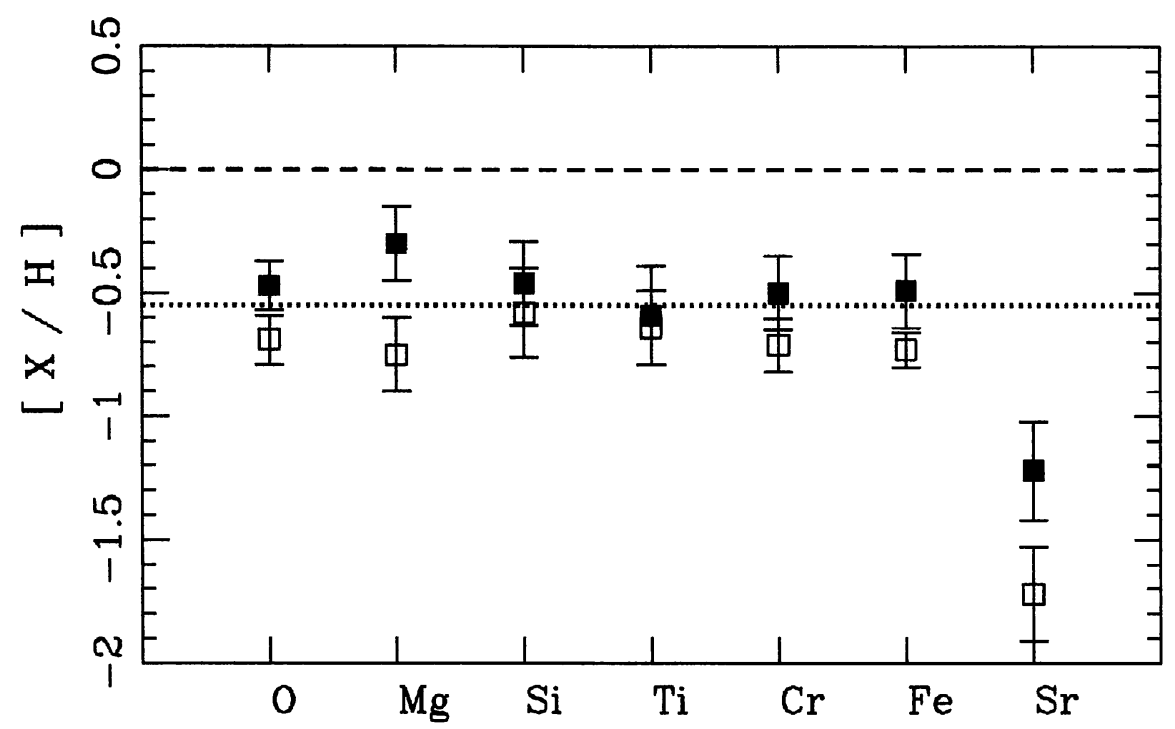

Figure 1. Average stellar abundances in NGC 6822 from three A-type supergiants (solid squares). For comparison, the nebular oxygen abundance is shown (dotted line, from Pagel et al. 1980), as well as solar (dashed line, from Grevesse \& Sauval 1998). Average abundances from ten SMC A-type supergiants are also shown (open squares from Venn 1999).

bursting SFH is nearly opposite, i.e., star formation occuring in the SMC during the hiatus in NGC 6822, and vice versa.

The question then is why do these two galaxies have the same $\alpha / \mathrm{Fe}$ ratios? Even if we take the SFHs as given, there are still several possibilities. Firstly, that the $\alpha / \mathrm{Fe}$ ratios primarily reflect the old and intermediate-aged populations, which have already pumped out most of their chemical elements. Thus, the recent burst in NGC 6822 is having very little effect either because the new elements are locked in a hot phase (e.g., as seen in NGC 1569 by Martin, Kobulnicky \& Heckman 2002) or because the percentage of new metals being added to the recently formed stars is very small (A-type supergiants typically have ages $<10 \mathrm{Myr}$ ). Unfortunately, these possibilities are harder to understand in the SMC where star formation has been ongoing (and possibly the dominant stellar population) for at least $4 \mathrm{Gyr}$, thus we might expect to see an enriched $\alpha / \mathrm{Fe}$ ratio. That gas is being drawn out now from the SMC through interactions, e.g., the Magellanic Bridge, does not seem to explain the loss of new metals since analysis of Bridge stars has suggested metallicities similar to or below that in the SMC (e.g., Rolleston et al. 1999).

Oxygen gradient? We have examined the oxygen abundances in NGC 6822 versus their galactocentric distances within that galaxy, using the $\mathrm{H}$ I major axis position angle and inclination from Brandenburg \& Skillman (1998). We find the stellar and nebular oxygen abundances are consistent with a mean underabundance of $\sim-0.5$ dex, but also a linear least-squares fit to the stellar data and nebular data where both $\mathrm{O}$ II and O III lines were detected suggests a gradient of $\sim-0.1 \mathrm{dex} / \mathrm{kpc}$ (see Figure 2). Such an abundance gradient would be similar to 


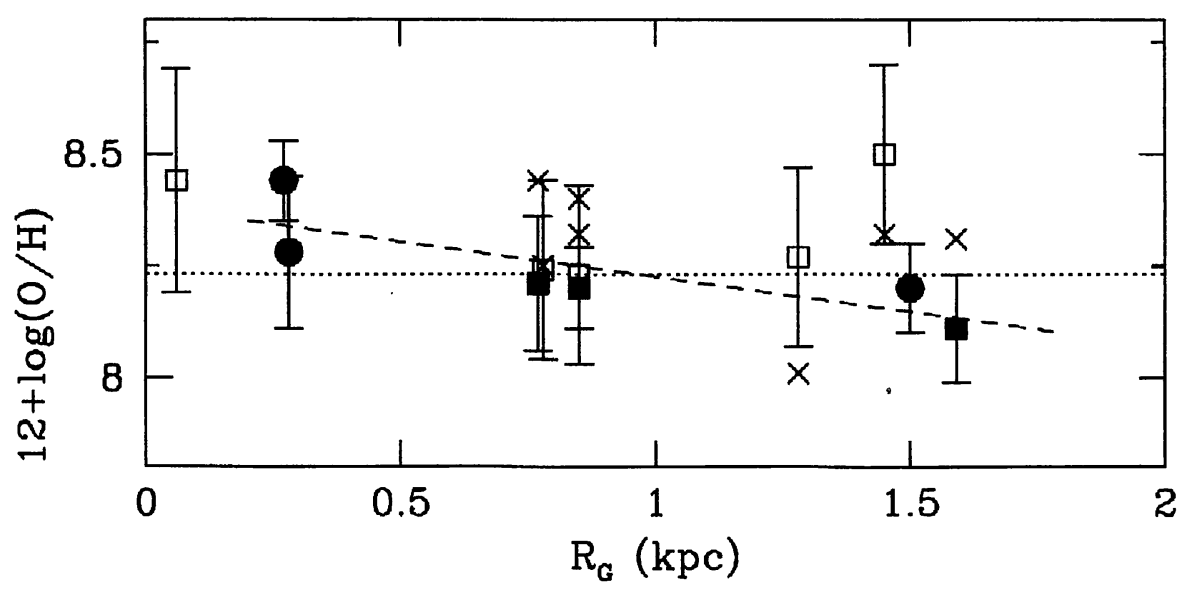

Figure 2. Stellar (filled circles) and nebular oxygen abundances in NGC 6822 showing that a linear least-squares fit (dashed line) suggests a galactic abundance gradient of $\sim-0.1 \mathrm{dex} / \mathrm{kpc}$. Nebular abundances are from Pagel et al. (1980, filled squares represent nebular abundances where both the $\mathrm{O}$ II and $\mathrm{O}$ III lines are detected, whereas hollow squares represent nebular abundances from O II alone). The mean nebular abundance is shown by the dotted line. In contrast, a re-determination of the nebular abundances by Pilyugin (2001) are marked by $\mathrm{X}$. Note that Pilyugin's mean abundance is $\sim 0.1 \mathrm{dex}$ higher.

that seen in the Galaxy (e.g., Smartt \& Rolleston 1997). It is difficult to imagine why this small dwarf irregular galaxy might not be well mixed over only a $2 \mathrm{kpc}$ radius. If confirmed by additional stellar and nebular measurements, this could provide a strong constraint on mixing timescales.

$S r$ Abundances? Finally, we note that the abundances of $\mathrm{Sr}$ (an $s \& r$-process element) are signficantly lower than the underabundances deduced from the $\alpha$ and iron-group elements in both NGC 6822 and the SMC. The $[\mathrm{Sr} / \mathrm{Fe}]$ ratios range from -0.5 to -1.5 dex. While a non-LTE effect may be indicated in the Galactic A-type supergiants, the effect appears to be smaller than the observed range ( $\leq 0.5 \mathrm{dex}$, Venn 1995). Thus, $\mathrm{Sr}$ does appear to be significantly underabundant in several of the dwarf irregular stars. In fact, the abundance distribution is reminiscent of the shot noise seen in metal-poor halo stars $([\mathrm{Fe} / \mathrm{H}]<-2.5$, see figure 9 by McWilliam 1997), where the dispersion reflects an inhomogeneous composition of the material due to SNII events. Could shot noise be affecting the $r$-process ratios in the stars in the dwarf irregulars at metallicities of $[\mathrm{Fe} / \mathrm{H}]>-1.0$ ? This would imply little $s$-process contribution to $\mathrm{Sr}$ from AGB stars. Could there be a difference in the $s$ or $r$-process yields in the dwarf irregulars? This would imply yields are somehow related to environment which does not seem likely. None of the possibilities are currently satisfactory, thus we shall attempt to observe additional $s \& r$-process elements in the future.

\section{WLM, Sextants A, and GR 8}

The remaining three dwarf irregular galaxies have larger distances from the Galaxy, thus even the brightest supergiants are quite faint. In WLM, we have 


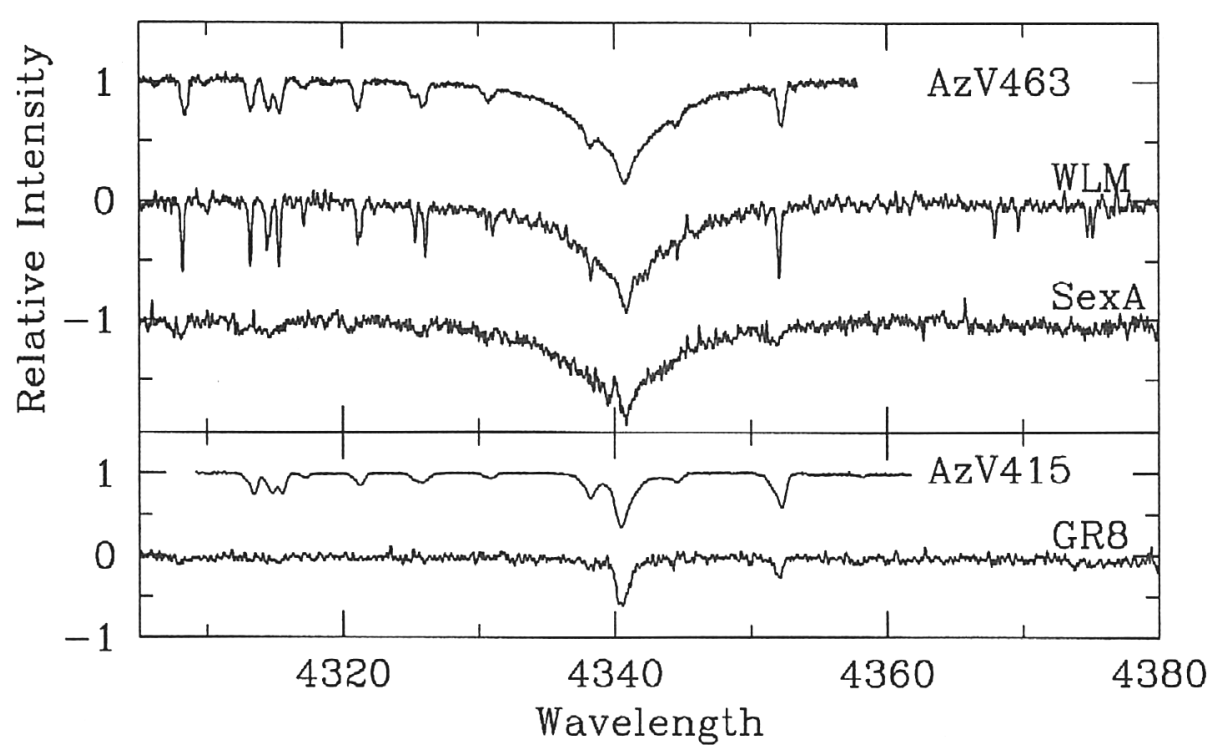

Figure 3. Sample spectra of stars in the SMC (top spectra in each panel, AzV463 and AzV415) and our targets in WLM, Sextants A, and GR 8. The top panel compares stars of similar atmospheric parameters (A7 Iab-Ib spectra). The bottom panel compares our GR 8 object to AzV 415, which has similar atmospheric parameters but is also a known LBV. We note that the objects in Sextants A and GR 8 are clearly more metal-poor than their SMC comparisons (examine the iron-group lines to the left and right of $\mathrm{H} \gamma$ ), however the WLM object has about the same metallicity as the SMC comparison.

obtained high quality spectra for two normal A-type supergiants with $V \simeq 17.9$ using the VLT-UVES in 3 to 5 hours. In Sextants A and GR 8, the brightest normal supergiant in each has $V \simeq 19.2$, and each have required 14 hours of observations with VLT-UVES. Sample spectra around the $\mathrm{H} \gamma$ Balmer line are shown in Figure 3. The Sextants A and GR 8 objects do indeed appear to be more metal-poor than their SMC comparison stars. This can be seen from an examination of the iron-group lines (Fe II, Sc II, Ti II, Cr II) to the left and right of the $\mathrm{H} \gamma$ Balmer line. Our preliminary analyses suggest $[\mathrm{Fe} / \mathrm{H}]=-1.0$ and -1.2 for GR 8 and Sex A, respectively. These are in good agreement with the nebular oxygen abundances (Skillman et al. 1989a,b; van Zee, Skillman \& Haynes 1999; Moles, Aparicio \& Masegosa 1990).

The same cannot be said for the WLM spectrum. In fact, we find that the WLM metallicity is quite similar to the SMC (the spectral lines are quite sharp, thus their equivalent widths are very close to those of the SMC comparison star). A second star in WLM (not shown in Figure 3) produces the same result. We find the mean metallicity in WLM from two A-type supergiants is $[\mathrm{Fe} / \mathrm{H}]=-0.6$. And, similar to the stars in NGC 6822 and the SMC, we find the stellar $\alpha / \mathrm{Fe}$ ratio to be solar, thus $[\mathrm{O} / \mathrm{H}] \simeq-0.6$ as well. In contrast, the nebular oxygen abundance for WLM is $[\mathrm{O} / \mathrm{H}]=-1.0$ (Skillman et al. 1989a; Hodge \& Miller 1995, determined from both O II and O III lines). While we still consider our 


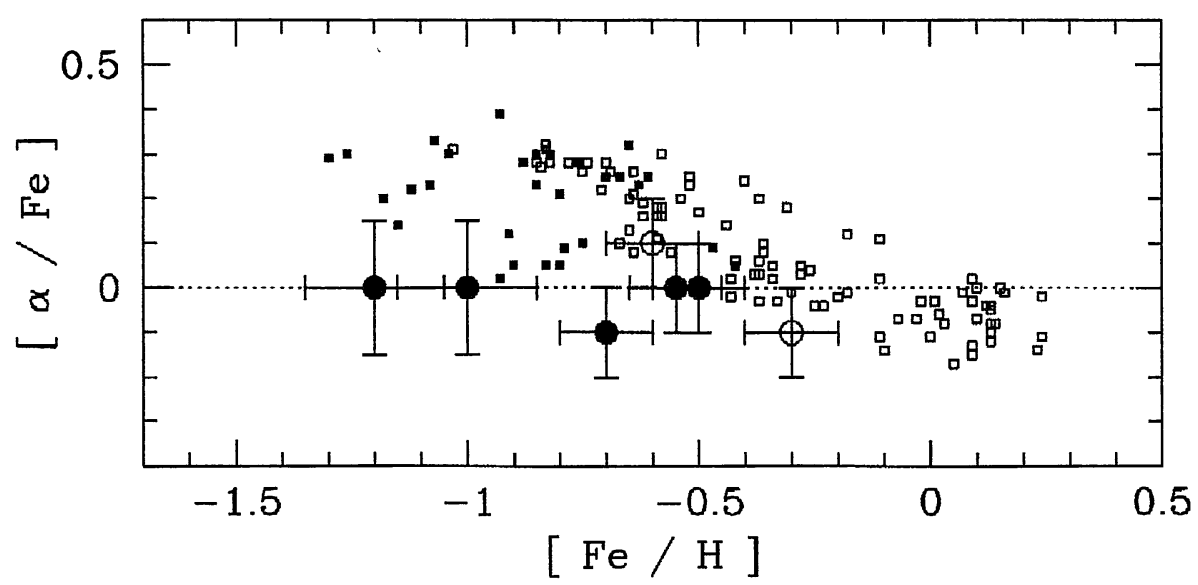

Figure 4. $[\alpha / \mathrm{Fe}]$ versus $[\mathrm{Fe} / \mathrm{H}]$ for stars in dwarf irregular galaxies (solid circles). The average of three stars in NGC 6822 is the highest metallicity dot, followed by two stars in WLM, then one star in GR 8 and the star in Sex A has the lowest metallicity. For comparison, the average abundances in the SMC and LMC are shown (open circles), as well as Galactic disk stars (open squares, Edvardsson et al. 1993) and metal-rich halo stars (filled squares, Nissen \& Schuster 1997).

results preliminary, we note that this is the first place where the A-supergiant and nebular oxygen abundances have not been in excellent agreement.

\section{1. $\alpha /$ Fe ratios in dwarf irregulars}

Our preliminary analyses of the spectra of stars in the dwarf irregulars have allowed us to calculate a mean $\alpha$-element abundance (an average of $\mathrm{O}, \mathrm{Mg}$, and $\mathrm{Si}$ ). A comparison of the ratios in these three dwarf irregular galaxies, NGC 6822, and the SMC is shown in Figure 4 where it can be seen they are all roughly solar.

Again, an examination of their estimated SFHs from interpretation of their color-magnitude diagrams (Mateo 1998) reveals significant differences, although they do have globally similar properties as well. For example, these three galaxies and NGC 6822 generally have had significant star formation in their past ( $>5$ Gyr ago), followed by a relative hiatus, followed by a very recent burst $(<1 \mathrm{Gyr})$. Since there has been enough time during the hiatus $(>1 \mathrm{Gyr})$, then one might expect $[\alpha / \mathrm{Fe}]$ abundances from the old population to have declined to the solar value (or even below, Gilmore \& Wyse 1991). Thus, as discussed for NGC 6822 above, perhaps the new elements from the recent burst have yet to be mixed into the warm ISM, perhaps the new elements are simply too small of a fraction of the total gas mass to significantly alter the $\alpha / \mathrm{Fe}$ ratios already attained, or possibly the recent bursts have produced solar ratios of $\alpha / \mathrm{Fe}$, particularly if they have lasted for $\sim 1$ Gyr already (the assumed timescale for the $\mathrm{SN}$ Ia). Whatever the reason, there is clearly something special about the solar $\alpha /$ Fe ratio. 
Acknowledgments. KAV would like to thank the National Science Foundation for funding through a CAREER/PECASE award, AST-9984073. Thanks to Christophe Pinte for work on the spectral analyses of the stars in Sextants A and GR 8. Thanks also to Macalester students Sonya Clarkson and Lissa Miller for measurements and work on the analyses of stars in WLM and NGC 6822.

\section{References}

Brandenburg, H.J., Skillman, E.D. 1998, A\&AS 193, 7011

Edvardsson, B., Andersen, J., Gustafsson, B., Lambert, D.L., Nissen, P.E., Tomkin, J. 1993, A\&A 275, 101

Gallart, C., Aparicio, A., Vílchez, J.M. 1996a, AJ 112, 1928

Gallart, C., Aparicio, A., Bertelli, G., Chiosi, C. 1996b, AJ 112, 2596

Gilmore, G., Wyse, R.F.G. 1991, Astrophys. Letters 367, 55

Grebel, E.K. 1999, in: P. Whitelock \& R. Cannon (eds.), The Stellar Content of Local Group Galaxies, Proc. IAU Symp. No. 192 (San Francisco: ASP), p. 17

Grevesse, N., Sauval, A.J. 1998, Space Sci. Reviews 85, 161

Hodge, P., Miller, B.W. 1995, ApJ 451, 176

Martin, C.L., Kobulnicky, H.A., Heckman, T.M. 2002, ApJ 574, 663

Mateo, M. 1998, Ann. Review Astron. Astrophys. 36, 435

McWilliam, A. 1997, Ann. Review Astron. Astrophys. 35, 503

Moles, M., Aparicio, A., Masegosa, J. 1990, A\&A 228, 310

Nissen, P.E., Schuster, W.J. 1997, A\&A 326, 751

Pagel, B.E.J., Tautvaišienè, G. 1998, MNRAS 299, 535

Pagel, B.E.J., Edmunds, M.G., Smith, G. 1980, MNRAS 193, 219

Pilyugin, L.S. 2001, A\&A 374, 412

Przybilla, N., Butler, K., Becker, S.R., Kudritzki, R.-P. 2001, A\&A 369, 1009

Rolleston, W.R.J., Dufton, P.L., McErlean, N.D., Venn, K.A. 1999, A\&A 348, 728

Skillman, E.D., Terlevich, R., Melnick, J. 1989a, MNRAS 240, 563

Skillman, E.D., Kennicutt, R.C., Hodge, P.W. 1989b, ApJ 347, 875

Smartt, S.J., Rolleston, W.R.J. 1997, ApJ 481, 47

Venn, K.A. 1995, ApJS 99, 659

Venn, K.A. 1999, ApJ 518, 405

Venn, K.A., Lennon, D.J., Kaufer, A., McCarthy, J.K., Przybilla, N., Kudritzki, R.-P., Lemke, M., Skillman, E.D., Smartt, S.J. 2001, ApJ 547, 765

van Zee, L., Skillman, E.D., Haynes, M.P. 1999, A\&AS 194, 504

\section{Discussion}

LEITHERER: Could the abundance patterns in these dwarf galaxies reflect the interaction history with the Milky Way, rather than mixing processes and stellar evolution? Surely this must affect the mass loss history of the ISM?

VENN: I would agree, and yet the Pagel \& Tautvaišienè (1998) models were calibrated to the present-day $\mathrm{O} / \mathrm{Fe}$ rates, and $d o$ a decent job explaining the existing abundance ratios.

SMITH: Concerning the star formation history in the SMC, there is some evidence that the clusters may indicate a smooth age-metalicity relation. Could this not suggest a 
smoother star formation history?

VENN: Yes. I have favored the bursting SFH based on it's dynamical-orbital parameters, i.e., knowing the SMC-LMC-Galaxy have been interacting, which could induce star formation bursts. But also the metallicites in the globular clusters are not easy to interpret since Mighell et al. (1998, AJ 116, 2395) find one thing and yet Da Costa \& Hatzidimitriou (1998, AJ 115, 1934) found another.

MATTEUCCI: I have a comment: there is no surprise in finding solar $\alpha / \mathrm{Fe}$ ratios in irregular galaxies, since it is predicted by the time-delay model of chemical evolution. In fact, objects with a global lower star formation rate than in the solar neighborhood are expected to reach a lower $[\mathrm{Fe} / \mathrm{H}]$ at the present time, but the contribution of type Ia Sne occurs always in the same timescale, with the consequence of having $[\alpha / \mathrm{Fe}] \approx 0$. Abundances are integral properties and depend more on the integrated star formation rate than on its precise temporal behaviour.

WALBORN: The $\mathrm{H} \alpha$ profile of the star in GR 8 does not necessarily imply that it is an LBV, because McCarthy, Kudritzki et al. found and explained similar profiles in Keck observations of very luminous A-type supergiants in M 33 .

VENN: Actually, one A-type supergiant in M 33 (117A) has a photospheric profile. The other (B234) is a candidate LBV. But I'm also suggesting that it may be an LBV based on the similar atmospheric parameters to $\mathrm{R} 40$, combined with the $\mathrm{H} \alpha$ profile.

KUDRITZKI: Refering to the question by Nolan Walborn before: well, Kim has, of course, provided us with $\mathrm{H} \alpha$ profiles of the GR 8 A-type supergiants and we are already working on the mass-loss determination as we did in our M31 and M 33 work.

You-HuA CHU: I have one comment about the chemical abundance evolution of the SMC. The SMC, LMC and Milky Way tidal interaction has drawn a large amount of gaseous mass out of the SMC. The mass loss of the SMC needs to be taken account in the chemical abundances evolution.

VEnN: That makes sense, and yet the Pagel \& Tautvaišienè (1998) closed box models do reproduce the abundance ratio observations rather well. 